Pacific Journal of Mathematics

REPRESENTATIONS OF WITT GROUPS 


\title{
REPRESENTATIONS OF WITT GROUPS
}

\author{
JoHn BRENdAN SUllivaN
}

This paper gives a tensor product theorem for the coordinate rings of the finite-dimensional Witt groups. This theorem leads to a demonstration of the equivalence of the representation theory of the Witt groups with that of certain truncated polynomial rings.

Introduction. The Steinberg tensor product theorem [1, Ch. A, §7] for a simply connected, semisimple algebraic group $G$ in characteristic $p$ displays irreducible $G$-modules as tensor products of Frobenius powers of infinitesimally irreducible $G$-modules (modules which are irreducible for the kernel $G^{1}$ of the Frobenius morphism of $G$ ).

A goal of modular representation theory is the expression of the coordinate ring of $G$ in terms of tensor products of Frobenius powers of $G$-modules which are suitably elementary for $G^{1}$. In this paper, we give a tensor product theorem for the finite-dimensional Witt groups. We produce a subcoalgebra $C$ of the coordinate ring $A$ of the $m$-dimensional Witt group $W_{m}$ which is isomorphic to the coordinate ring of the kernel $W_{m}^{1}$ of the Frobenius morphism. $A$ is the inductive limit of tensor products of Frobenius powers of $C$ $[\S 3$, Theorem].

One can see some things about the representations of $W_{m}$. First, every finite-dimensional representation of $W_{m}^{1}$ extends to a representation of $W_{m}$ on the same representation space [\$5]. Second, a representation of $W_{m}$ on a finite-dimensional vector space $V$ is determined by a family $\left\{f_{1}, \cdots, f_{n}\right\}$ of commuting endomorphisms of $V$ such that $f_{i}^{p m}=0$. In other words, the representations of $W_{m}$ on $V$ may be studied via the representations of the algebras $\left\{k\left[x_{1}, \cdots, x_{n}\right] /\left(x_{1}^{p^{m}}, \cdots, x_{n}^{p^{m}}\right)\right\}_{n}$ on $V$ [Theorem, $\left.\S 4\right]$. In particular, the representations of $W_{m}$ which correspond to the representations of $k\left[x_{1}\right] /\left(x_{1}^{p^{m}}\right)$ give canonical extensions for the representations of $W_{m}^{1}$.

This linear formulation of the representation theory of $W_{m}$ leaves one with the apparently difficult problem of determining the representation theory of $k\left[x_{1}, \cdots, x_{n}\right] /\left(x_{1}^{p^{m}}, \cdots, x_{n}^{p^{m}}\right)$.

For the definition of the Witt groups, see [2, Ch. 5, §1].

Notation. Let $A$ denote the coordinate ring of the $m$-dimensional Witt group $W_{m}$, as a reduced, connected group scheme over the prime field $k=F_{p}$. For any subcoalgebra $C$ of $A$ which contains $k$, let $C^{\left(p^{i}\right)}$ be the image of $C$ under the $i$ th-power of the Frobenius 
morphism of $A$. We may form the inductive family of coalgebras $\left\{C \otimes C^{(p)} \otimes \cdots \otimes C^{\left(p^{n}\right)}\right\}_{n=0}^{\infty}$, where $C \otimes \cdots \otimes C^{\left(p^{n}\right)} \hookrightarrow C \otimes \cdots \otimes C^{\left(p^{n}\right)} \otimes$ $C^{\left(p^{n+1}\right)}$ is the canonical morphism onto $C \otimes \cdots \otimes C^{\left(p^{n}\right)} \otimes k$. Let $\underset{n}{\lim } C \otimes C^{(p)} \otimes \cdots \otimes C^{\left(p^{n}\right)}$ be the coalgebra inductive limit of the family.

Let $\Pi: A \rightarrow A / M^{(p)} A$ be the quotient morphism, where $M^{(p)}$ is the image of the augmentation ideal $M$ under the Frobenius morphism. We show in $\S 3$ that there is a coalgebra splitting $s: A / M^{(p)} A \rightarrow A$ of $\Pi$ such that $A$, as a coalgebra, is isomorphic to $\underset{n}{\lim } C \otimes C^{(p)} \otimes$ $\cdots \otimes C^{\left(p^{n}\right)}$ where $C=$ image $s$.

o. We require some facts from [3, Def. 6] of $K$. Newman. Let $W_{m+1}$ be the $(m+1)$-dimensional Witt group over $k=F_{p}$, with coordinate ring $A_{m+1}$. As an algebra, $A_{m+1}$ is the polynomial ring $k\left[X_{1}, X_{p}, X_{p^{2}}, \cdots, X_{p^{m}}\right]$ on $(m+1)$-variables. Grade $A_{m+1}$ by letting $X_{p^{i}}$ have degree $p^{i}$. The coproduct $\Delta$ of $A_{m+1}$ is the following: $\Delta X_{p^{i}}=\sum_{j=0}^{p^{i}} Q_{j} \otimes Q_{p^{i}-j}$, where $Q_{j}$ is a homogeneous (relative to the grading) polynomial of degree $j$. In particular, $Q_{0}=1, Q_{p^{i}}=X_{p^{i}}$ and $\left\{Q_{j}\right\}_{j=0}^{p m}$ is a sequence of divided powers.

Since degree $Q_{j}=j, Q_{j}$ lies in $k\left[X_{1}, X_{p}, \cdots, X_{p m-1}\right]$ for $j<p^{m}$. The coordinate ring $A$ of $W_{m}$ may be identified with the sub-Hopf algebra $k\left[X_{1}, X_{p}, \cdots, X_{p^{m-1}}\right]$ of $A_{m+1}$.

1. The coalgebra splitting of $\Pi$. $M=\left(X_{1}, X_{p}, \cdots, X_{p^{m-1}}\right)$ is the augmentation ideal of $A$. Let $C$ be the $k$-span of $\left\{Q_{j}\right\}_{j=0}^{p_{m-1}}$. $C$ is an irreducible coalgebra of dimension $p^{m}$, with $k \cdot X_{1}$ as its space of primitive elements. Since the coalgebra map $f: C \hookrightarrow A \stackrel{\Pi}{\rightarrow} A / M^{(p)} A$ has an injective restriction to $k \cdot X_{1}, f$ is injective [5, Lemma 11.0.1]. Since $\left(A / M^{(p)} A\right)^{*}$ is the restricted universal enveloping algebra of $\left(M / M^{2}\right)^{*}[3,13.2 .3], \operatorname{dim}_{l}\left(A / M^{(p)} A\right)^{*}=p^{\mathrm{dim}_{l}\left(M / M^{2}\right)^{*}}=p^{m}$. Therefore, $\operatorname{dim}_{k}\left(A / M^{(p)} A\right)=p^{m}$ and $f$ is an isomorphism. $s=f^{-1}$ is the coalgebra splitting of $\Pi$ that we use.

2. The value of $\Pi$ at $Q_{j}$. Let $0 \leqq j<p^{m}$. Write $j=\sum_{i=0}^{m-1} a_{i} p^{i}$ where $0 \leqq a_{i}<p$.

LEMMA. $\Pi\left(Q_{j}\right)$ is a nonzero scalar multiple of $\Pi\left(X_{1}^{a_{0}} X_{p}^{a_{1}} \cdots X_{p^{m-1}}^{a_{m-1}}\right)$.

Proof. $Q_{j}$ is a linear combination of elements $X_{1}^{b_{0}} X_{p}^{b_{1}} \cdots X_{p m-1}^{b_{m}=1}$ where $\sum b_{i} p^{i}=j$ by $\S 0$. If $\left\{b_{i}\right\}_{i} \neq\left\{a_{i}\right\}_{i}$, then $b_{i} \geqq p$ for some $i$, and $\Pi\left(X_{1}^{b_{0}} X_{p}^{b_{1}} \cdots X_{p^{m-1}}^{b_{m-1}}\right)=0$. Therefore, $\Pi\left(Q_{j}\right) \in k \cdot \Pi\left(X_{1}^{a_{0}} X_{p}^{a_{1}} \cdots X_{p_{m-1}}^{a_{m-1}}\right)$, where the coefficient of $\Pi\left(X_{1}^{a_{0}} X_{p}^{a_{1}} \cdots X_{p m-1}^{a_{m-1}}\right)$ is nonzero since the map 
$f$ of $\S 1$ is injective.

3. The coalgebra structure of the coordinate ring. Give the set of monomials in $A$ the reverse lexicographic total order: $X_{1}^{a_{0}} X_{p}^{a_{1}} \cdots X_{p^{m}=1}^{a_{m-1}}>X_{1}^{b_{0}} X_{p}^{b_{1}} \cdots X_{p^{m=1}}^{b_{m-1}}$ if there is an index $k$ such that $a_{k}>b_{k}$ and $a_{i}=b_{i}$ for $i>k$.

Let $\left\{a_{i}\right\}_{0}^{m-1}$ be a sequence where $0 \leqq a_{i}<p$, and let $\left\{b_{i}\right\}_{0}^{m-1}$ be a different sequence, where $0 \leqq b_{i}$.

LeMmA. If $\sum_{i=0}^{m-1} a_{i} p^{i}=\sum_{i=0}^{m-1} b_{i} p^{i}$, then $\quad X_{1}^{a_{0}} X_{p}^{a_{1}} \cdots X_{p^{m-1}}^{a_{m-1}}>$ $X_{1}^{b_{0}} X_{p}^{b_{1}} \cdots X_{p^{m-1}}^{b_{m-1}}$.

Proof. Let $k$ be the maximal index such that $a_{k} \neq b_{k}$. If $b_{k}>a_{k}$, then $\sum_{i=0}^{m-1} b_{i} p^{i}>\sum_{i=0}^{m-1} a_{i} p^{i}$ since $a_{i}<p$. Therefore, we must have $a_{k}>b_{k}$ and $X_{1}^{a_{0}} \cdots X_{p^{m-1}}^{a_{m-1}}>X_{1}^{b_{0}} \cdots X_{p^{m-1}}^{b_{m-1}}$.

Let $C$ be the coalgebra formed in $\S 1$.

THEOREM. The map $\underset{n}{\stackrel{\lim }{\longrightarrow}} C \otimes C^{(p)} \otimes \cdots \otimes C^{\left(p^{n}\right)} \rightarrow A$, induced by multiplication; $C \otimes C^{(p)} \otimes \cdots \otimes C^{\left(p^{n}\right)} \rightarrow A$, is an isomorphism of coalgebras.

Proof. Denote the map by $g$.

Surjectivity of $g$. Suppose that monomials $X_{1}^{b_{0}} X_{p}^{b_{1}} \cdots X_{p^{m-1}}^{b_{m-1}}$ less than $X_{1}^{a_{0}} X_{p}^{a_{1}} \cdots X_{p^{m-1}}^{a m-1}$ in the ordering lie in the image of $g$. We show that $X_{1}^{a_{0}} X_{p}^{a_{1}} \cdots X_{p m-1}^{a_{m-1}}$ also lies there.

Write $a_{i}=\sum_{j} a_{i j} p^{j}$, where $0 \leqq a_{i j}<p$. Let $t_{k}=\sum_{i=0}^{m-1} a_{i k} p^{i}$. By the lemmas of $\S 2$ and $\S 3$,

$$
Q_{t_{k}}=U_{k} \cdot X_{1}^{a_{0 k}} X_{p}^{a_{1 k}} \cdots X_{p^{m-1}}^{a_{m-1, k}}+Y_{k},
$$

where $Y_{k}$ is a linear combination of monomials of degree $t_{k}$ and less than $X_{1}^{a_{0 k}} \cdots X_{p^{m-1}}^{a_{m-1, k}}$ in the ordering, and where $U_{k}$ is a nonzero scalar. Therefore,

$$
\prod_{k=0}^{m=1} Q_{t_{k}}^{p^{k}}=\prod_{k=0}^{m-1} U_{k}^{p^{k}} \cdot X_{1}^{a_{0}} X_{p}^{a_{1}} \cdots X_{p^{m-1}}^{a_{m-1}}+Y,
$$

where $Y$ is a linear combination of monomials which are less than $X_{1}^{a_{0}} X_{p}^{a_{1}} \cdots X_{p^{m-1}}^{a_{m-1}}$. Since $\prod_{k=0}^{m-1} Q_{t_{k}}^{p^{k}}$ and $Y$ lie in the image of $g$, so does $X_{1}^{a_{0}} X_{p}^{a_{1}} \cdots X_{p^{m-1}}^{a_{m-1}}$.

Injectivity of $g$. Since $g$ is surjective, so is $\Pi \circ g: \underset{n}{\longrightarrow} C \otimes$ $C^{(p)} \otimes \cdots \otimes C^{\left(p^{n}\right)} \stackrel{g}{\rightarrow} A \stackrel{[\boldsymbol{I I} !}{\rightarrow} A / M^{\left(p^{t}\right)} A$ for any $t ;$ at the same time, ${ }^{n} C^{\left(p^{j}\right)} \hookrightarrow$ $A \stackrel{\Pi}{\rightarrow} A / M^{\left(p^{t}\right)} A$ has image $=k$ if $j \geqq t . \quad$ Therefore, $C \otimes C^{(p)} \otimes \cdots \otimes$ $C^{\left(p^{t-1}\right)} \stackrel{\text { mult. }}{\longrightarrow} A \stackrel{\Pi}{\rightarrow} A / M^{\left(p^{t}\right)} A$ is surjective. Since $\operatorname{dim}_{k}\left(A / M^{\left(p^{t}\right)} A\right)=p^{m t}$ 
by [4] or by inspection, and $\operatorname{dim}_{k}\left(C \otimes C^{(p)} \otimes \cdots \otimes C^{(p t-1)}\right)=p^{m t}$, $\Pi \circ$ mult. is an isomorphism of coalgebras. In particular, $C \otimes$ $C^{(p)} \otimes \cdots \otimes C^{\left(p^{t-1}\right)} \stackrel{\text { mult. }}{\longrightarrow} A$ is injective. Hence, $g$ is injective.

4. Representation theory of $W_{m}$. The dual algebra $U=$ $\left(A / M^{(p)} A\right)^{*}$ is the restricted universal enveloping algebra of the abelian $p$-Lie algebra $L=\left(M / M^{2}\right)^{*}[5,13.2 .3]$.

Lemma. There is a k-basis $f_{0}, \cdots, f_{m-1}$ for $L$, where $f_{i}^{p}=f_{i+1}$ for $i<m-1$ and $f_{m-1}^{p}=0$.

Proof. Define $f_{j}$ on the $k$-basis $X_{1}, X_{p}, \cdots, X_{p^{m-1}}$ for $M / M^{2}$ by $f_{j}\left(X_{p i}\right)=\delta_{i j}$. We have the following to complete the proof.

(1) If $i \neq j+1$, then $f_{j}^{p}\left(X_{p^{i}}\right)=\left(\bigotimes^{p} f_{j}\right)\left(\Delta^{p-1} X_{p^{i}}\right)$ is 0 , since $\Delta^{p-1} X_{p^{i}}$ is homogeneous of degree $p^{i}$ under the grading of $\otimes^{p} A$ induced from the grading of $A$, while $\otimes^{p} f_{j}$ can be nonzero only at monomials in $\otimes^{p} A$ of degree $p^{j+1}$.

(2) One may check that $f_{j}^{p}\left(X_{p^{j+1}}\right)=1$.

To proof is complete.

By this lemma, the algebra map from the polynomial ring $k[f]$ to $U$ mapping $f$ to $f_{1}$ induces an isomorphism of $k$-algebras $k[f] /\left(f^{p^{m}}\right) \cong U$.

Denote by $R_{n}$ the set of isomorphism classes of finite-dimensional representations of $W_{m}$ whose coefficients lie in $C \otimes C^{(p)} \otimes \cdots \otimes$ $C^{\left(p^{n}\right)} \hookrightarrow A$. The canonical map $C \otimes C^{(p)} \otimes \cdots \otimes C^{\left(p^{n}\right)} \hookrightarrow C \otimes C^{(p)} \otimes \cdots \otimes$ $C^{\left(p^{n}\right)} \otimes C^{\left(p^{n+1}\right)}$ induces $R_{n} \hookrightarrow R_{n+1}$. Then $R=\mathrm{U}_{n} R_{n}$ is the set of isomorphism classes of finite-dimensional representations of $W_{m}$.

Let $B$ denote the quotient of the polynomial ring $F_{p}\left[X_{0}, \cdots, X_{n}, \cdots\right]$ on generators $\left\{X_{i}\right\}_{i=0}^{\infty}$ by the ideal $\left(X_{0}^{p^{m}}, \cdots, X_{n}^{p^{m}}, \cdots\right)$. Denote by $\widehat{B}$ the set of isomorphism classes among those finite-dimensional representations of $B$ in which all but a finite number of the $X_{i}$ act as the zero endomorphism. Denote by $\hat{B}_{n}$ the set of isomorphism classes of finite-dimensional representations of $k\left[X_{0}, \cdots, X_{n}\right] /\left(X_{0}^{p^{m}}, \cdots, X_{n}^{p^{m}}\right)$. The $\operatorname{map} k\left[X_{0}, \cdots, X_{n}, \cdots\right] /\left(X_{0}^{p^{m}}, \cdots, X_{n}^{p^{m}}, \cdots\right) \rightarrow k\left[X_{0}, \cdots, X_{n}\right] /\left(X_{0}^{p^{m}}, \cdots, X_{n}^{p^{m}}\right)$, $X_{i} \mapsto X_{i}$ for $i \leqq n$ and $X_{i} \mapsto 0$ for $i>n$, induces $\hat{B}_{n} \hookrightarrow \widehat{B}$, and $\hat{B}=$ $\mathbf{U}_{n} \hat{B}_{n}$.

THEOREM. There is a canonical bijection $R \rightarrow \hat{B}$, under which $R_{n}$ and $\widehat{B}_{n}$ correspond.

Proof. Since $C \cong A / M^{(p)} A$ as coalgebras, $C^{*} \cong U$ as algebras. Since $A$ is reduced, the Frobenius morphism on $A$ is injective, and $C \cong C^{\left(p^{i}\right)}$. Therefore, 
(1) $\left(C \otimes C^{(p)} \otimes \cdots \otimes C^{\left(p^{n}\right)}\right)^{*} \cong \otimes^{n+1} U \cong k\left[X_{0}, \cdots, X_{n}\right] /\left(X_{0}^{p^{m}}, \cdots, X_{n}^{p^{m}}\right)$. The first isomorphism is induced by the maps $U \rightarrow\left(C \otimes C^{(p)} \otimes \cdots \otimes C^{\left(p^{n}\right)}\right)^{*}$ which are dual to the maps $C \otimes C^{(p)} \otimes \cdots \otimes C^{(p)} \stackrel{\varepsilon_{0} \otimes \cdots \otimes \varepsilon_{i-1} \otimes I \otimes \varepsilon_{i+1} \otimes \cdots \otimes \varepsilon_{n}}{\longrightarrow}$ $C^{\left(p^{i}\right)}$, where $\varepsilon_{j}$ is the counit of $C^{\left(p^{j}\right)}$; the second isomorphism is induced by $X_{i} \mapsto 1_{0} \otimes \cdots \otimes 1_{i-1} \otimes f_{1} \otimes 1_{i+1} \otimes \cdots \otimes 1_{n}$, where $1_{j}$ is the identity of $U_{j}$. Here $u_{j}$ is the $j$ th copy of $u$ in $\otimes^{n+1} u$. Moreover,

(2) under dualization, the canonical map $C \otimes C^{(p)} \otimes \cdots \otimes C^{\left(p^{n}\right)} \hookrightarrow$ $C \otimes C^{(p)} \otimes \cdots \otimes C^{\left(p^{n+1}\right)}$ yields the map $k\left[f_{0}, \cdots, f_{n+1}\right] /\left(f_{0}^{p^{m}}, \cdots, f_{n+1}^{p^{m}}\right) \rightarrow$ $k\left[X_{0}, \cdots, X_{n}\right] /\left(X_{0}^{p^{m}}, \cdots, X_{n}^{p^{m}}\right)$ where $X_{i} \mapsto X_{i}$ for $i \leqq n$ and $X_{n+1} \mapsto 0$. The isomorphism $\left(C \otimes C^{(p)} \otimes \cdots \otimes C^{\left(p^{n}\right)}\right)^{*} \cong k\left[X_{0}, \cdots, X_{n}\right] /\left(X_{0}^{p^{m}}, \cdots, X_{n}^{p^{m}}\right)$ of (1) induces a bijection $R_{n} \rightarrow \hat{B}_{n}$ such that $\underset{R_{n+1}}{R_{n}} \rightarrow{\stackrel{B_{n+1}}{f}}_{R_{n+1}}^{\rightarrow}$ commutes by (2). Therefore, $R \stackrel{\sim}{\rightarrow} \hat{B}$.

5. Representations of $W_{m}^{1}$. The coalgebra $C$ constructed in $\S 1$ is isomorphic to the coordinate ring $A / M^{(p)} A$ of $W_{m}^{1}$ under the mapping $\pi: A \rightarrow A / M^{(p)} A$ restricted to $C$. Therefore, the representations of $W_{m}$ with coefficients in $C$ correspond to the representations of $W_{m}^{1}$ via the isomorphism between the coefficient coalgebras $C$ and $A / M^{(p)} A$, and very finite-dimensional representation of $W_{m}^{1}$ extends to a representation of $W_{m}$ on the same representation space.

\section{REFERENCES}

1. A. Borel, et al., Seminar on Algebraic Groups and Related Finite Groups, Lecture Notes in Mathematics, 131, Springer-Verlag, 1970.

2. M. Demazure and P. Gabriel, Groups Algebriques, Masson et Cie, Paris; North Holland, Amsterdam, 1970.

3. K. Newman, Constructing sequences of divided powers, Proc. Amer. Math. Soc., 31 (1972), 32-38.

4. J. Sullivan, Representations of the hyperalgebra of an algebraic group, Amer. J. Math., 100 (1978).

5. M. E. Sweedler, Hopf Algebras, Benjamin, New York, 1969.

Received May 7, 1977. Supported in part by a National Science Foundation Grant.

UNIVERSITY OF WASHINGTON

Seattle, WA 98195 



\title{
PACIFIC JOURNAL OF MATHEMATICS
}

\section{EDITORS}

RICHARD ARENS (Managing Editor)

University of California

Los Angeles, CA 90024

Charles W. Curtis

University of Oregon

Eugene, OR 97403

C. C. MOORE

University of California

Berkeley, CA 94720

\section{J. DUGUNDJI}

Department of Mathematics University of Southern California Los Angeles, CA 90007

R. Finn and J. Milgram

Stanford University

Stanford, CA 94305

\section{ASSOCIATE EDITORS}
E. F. BECKENBACH
B. H. NeumanN
F. WOLF
K. YOSHIDA

\section{SUPPORTING INSTITUTIONS}

\author{
UNIVERSITY OF BRITISH COLUMBIA \\ CALIFORNIA INSTITUTE OF TECHNOLOGY \\ UNIVERSITY OF CALIFORNIA \\ MONTANA STATE UNIVERSITY \\ UNIVERSITY OF NEVADA, RENO \\ NEW MEXICO STATE UNIVERSITY \\ OREGON STATE UNIVERSITY \\ UNIVERSITY OF OREGON
}

\author{
UNIVERSITY OF SOUTHERN CALIFORNIA \\ STANFORD UNIVERSITY \\ UNIVERSITY OF HAWAII \\ UNIVERSITY OF TOKYO \\ UNIVERSITY OF UTAH \\ WASHINGTON STATE UNIVERSITY \\ UNIVERSITY OF WASHINGTON
}

The Supporting Institutions listed above contribute to the cost of publication of this Journal, but they are not owners or publishers and have no responsibility for its content or policies.

Mathematical papers intended for publication in the Pacific Journal of Mathematics should be in typed form or offset-reproduced, (not dittoed), double spaced with large margins. Please do not use built up fractions in the text of the manuscript. However, you may use them in the displayed equations. Underline Greek letters in red, German in green, and script in blue. The first paragraph or two must be capable of being used separately as a synopsis of the entire paper. Items of the bibliography should not be cited there unless absolutely necessary, in which case they must be identified by author and journal, rather than by item number. Manuscripts, in triplicate, may be sent to any one of the editors. Please classify according to the scheme of Math. Reviews, Index to Vol. 39. All other communications should be addressed to the managing editor, or Elaine Barth, University of California, Los Angeles, California, 90024.

50 reprints to each author are provided free for each article, only if page charges have been substantially paid. Additional copies may be obtained at cost in multiples of 50 .

The Pacific Journal of Mathematics is issued monthly as of January 1966. Regular subscription rate: $\$ 72.00$ a year (6 Vols., 12 issues). Special rate: $\$ 36.00$ a year to individual members of supporting institutions.

Subscriptions, orders for numbers issued in the last three calendar years, and changes of address should be sent to Pacific Journal of Mathematics, P.O. Box 969, Carmel Valley, CA 93924, U.S.A. Older back numbers obtainable from Kraus Periodicals Co., Route 100, Millwood, NY 10546.

PUBLISHED BY PACIFIC JOURNAL OF MATHEMATICS, A NON-PROFIT CORPORATION

Printed at Kokusai Bunken Insatsusha (International Academic Printing Co., Ltd.). 8-8, 3-chome, Takadanobaba, Shinjuku-ku, Tokyo 160, Japan.

Copyright (C) 1978 by Pacific Journal of Mathematics Manufactured and first issued in Japan 


\section{Pacific Journal of Mathematics \\ Vol. 78, No. $2 \quad$ April, 1978}

Su-Shing Chen, Weak rigidity of compact negatively curved manifolds .... 273

Heinz Otto Cordes and D. A. Williams, An algebra of pseudodifferential operators with nonsmooth symbol ....................... 279

Herbert Paul Halpern, Normal expectations and integral decomposition of type III von Neumann algebras ......................... 291

G. Hochschild, On representing analytic groups with their

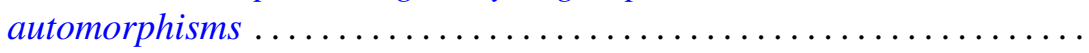

Dean G. Hoffman and David Anthony Klarner, Sets of integers closed under affine operators - the closure of finite sets ....................

Simeon Ivanov, On holomorphic relative inverses of operator-valued

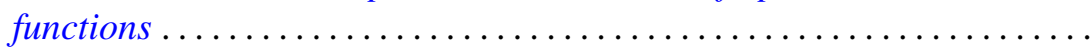

O. P. Juneja and M. L. Mogra, Radii of convexity for certain classes of

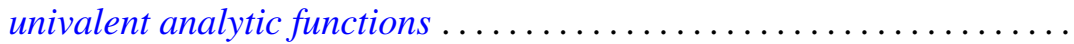

Hadi Kharaghani, The evolution of bounded linear functionals with application to invariant means.......................... 369

Jack W. Macki, A singular nonlinear boundary value problem .......... 375

A. W. Mason and Walter Wilson Stothers, Remarks on a theorem of $L$. Greenberg on the modular group ........................ 385

Kevin Mor McCrimmon, Peirce ideals in Jordan algebras . . . . . . . . . . . 397

John C. Morgan, II, On the absolute Baire property ................ 415

Gerard J. Murphy, Commutative non-Archimedean $C^{*}$-algebras ...

Masafumi Okumura, Submanifolds with L-flat normal connection of the

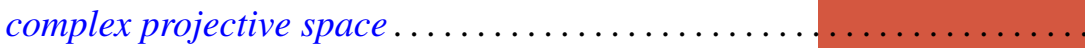

Chull Park and David Lee Skoug, Distribution estimates of barrier-crossing probabilities of the Yeh-Wiener process ...............

Irving Reiner, Invariants of integral representations ........

Phillip Schultz, The typeset and cotypeset of a rank 2 abelian group ..... 503

John Brendan Sullivan, Representations of Witt groups ....

Chia-Chi Tung, Equidistribution theory in higher dimensions . . . 